\title{
Electron microscopic evidence of charge-ordered bi-stripe structures in the bilayered colossal magnetoresistive manganite $\mathrm{La}_{2-2 x} \mathrm{Sr}_{1+2 x} \mathrm{Mn}_{2} \mathrm{O}_{7}$
}

\author{
Z. P. Luo* \\ Microscopy and Imaging Center, Biological Sciences Building West, Texas A\&M University, College Station, Texas 77843-2257, USA
}

D. J. Miller and J. F. Mitchell

Materials Science Division, Argonne National Laboratory, Argonne, Illinois 60439, USA

(Received 26 May 2004; revised manuscript received 28 October 2004; published 13 January 2005)

\begin{abstract}
In sharp contrast to the three-dimensional perovskite manganite, evidence of charge-ordered structures with bi-stripe models has been found on the bilayered manganite $\mathrm{La}_{2-2 x} \mathrm{Sr}_{1+2 x} \mathrm{Mn}_{2} \mathrm{O}_{7}$ based on in-situ electron microscopic studies. At the electronic doping level of $x=0.6$, a new face-centered charge-ordered superstructure was observed. This structure is composed of bi-stripes of $\mathrm{Mn}^{3+} \mathrm{O}_{6}$ and paired $\mathrm{Mn}^{4+} \mathrm{O}_{6}$ rows alternatively stacking along the ordering direction, which is assembled from the building blocks of the charge-ordered phase at $x=0.5$. Taking into account the systematic absence of reflections with the face-centered symmetry, its modulation vector was deduced as $\mathbf{q}=(1 / 10,1 / 10,0)$. Moreover, for another charge-ordered phase identified at $x=0.67$ with $\mathbf{q}=(1 / 6,1 / 6,0)$, again only the bi-stripe model fits the existing data over the Wigner-crystal model.
\end{abstract}

DOI: 10.1103/PhysRevB.71.014418

PACS number(s): 75.25.+z, 61.14.Lj

\section{INTRODUCTION}

The colossal magnetoresistive (CMR) phenomena in mixed-valent manganite perovskite materials stem from the interplay among the charge, spin and lattice degrees. ${ }^{1}$ To understand the physical properties of these materials, identification of the charge-ordered (CO) structure is crucial. At the half electronic hole doping level $x=0.5$ with the equal amount of $\mathrm{Mn}^{3+}$ and $\mathrm{Mn}^{4+}$, as predicted by Goodenough a half century ago, ${ }^{1}$ the $\mathrm{CO}$ structure is formed by the alternative distribution of the $\mathrm{Mn}^{3+} 3 d_{3 z^{2}-r^{2}}$ orbitals on the $\mathrm{Mn}$ sites, which results superlattice reflections predominantly from the cooperative Jahn-Teller distortions. ${ }^{2}$ However, when the doping level deviates away from the half doping level, significant controversies have arisen regarding the $\mathrm{CO}$ structures. Early work by Mori et al. ${ }^{3-5}$ proposed a $\mathrm{CO}$ structure of the three-dimensional (3D) perovskite $\mathrm{La}_{0.33} \mathrm{Ca}_{0.67} \mathrm{MnO}_{3}$ that has double $\mathrm{Mn}^{3+} \mathrm{O}_{6}$ octahedron stripes among the $\mathrm{Mn}^{4+} \mathrm{O}_{6}$ octahedron rows, the so-called bi-stripe model in the literature. In controversy, Fernández-Díaz et al. ${ }^{6}$ and Radaelli et al. ${ }^{7}$ pointed out that when single $\mathrm{Mn}^{3+} \mathrm{O}_{6}$ rows are separated as far apart as possible, the so-called Wigner-crystal model as the Coulomb repulsion energy can be minimized within this plane, ${ }^{8,9}$ better fits to their $\mathrm{x}$-ray and neutron diffraction data were obtained. Electron microscopic studies also supported the Wigner-crystal model over the bi-stripe model for this 3D perovskite. ${ }^{10}$ In this work on the study of naturally layered CMR compounds $\mathrm{La}_{2-2 x} \mathrm{Sr}_{1+2 x} \mathrm{Mn}_{2} \mathrm{O}_{7}$, discriminating of the different $\mathrm{CO}$ structures is made using in-situ electron microscopy combined with quantitative simulations of electron diffraction (ED) patterns and high-resolution electron microscopy (HREM) images based on the dynamic-diffraction theory. Our results show that for both two charge-ordered phases, identified at $x=0.6$ and $x=0.67$, respectively, in sharp contrast to the 3D perovskite that the Wigner-crystal model was suggested, ${ }^{6,7,9,10}$ only the bi-stripe models fit the experimental data surprisingly.
In the naturally layered compounds $\mathrm{La}_{2-2 x} \mathrm{Sr}_{1+2 x} \mathrm{Mn}_{2} \mathrm{O}_{7}$, since the double $\mathrm{MnO}_{2}$ sheets are separated by rock-salt-type blocking layers $(\mathrm{La}, \mathrm{Sr})_{2} \mathrm{O}_{2}$ [see Fig. 1(a) for $x=0.5$ ], these manganites provide opportunities to study not only the charge and spin interplay in reduced 2-dimensions, but also explore new phenomena that are not found in the 3D perovskite manganites. Therefore, they have attracted considerable attention in recent years. ${ }^{11-24}$ In the range of $0.32<x$ $<0.40$, a ferromagnetic metallic (FM) state was found,

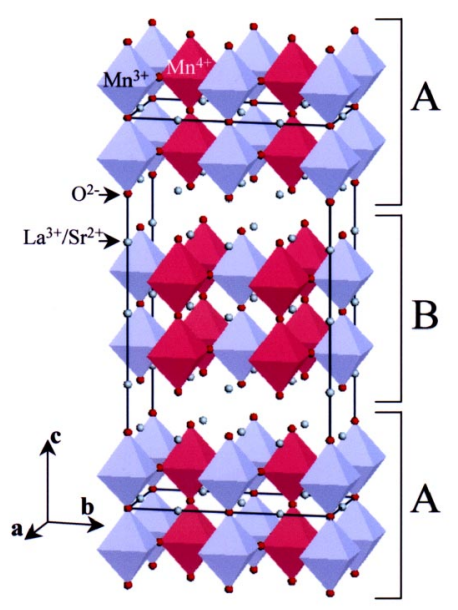

(a)

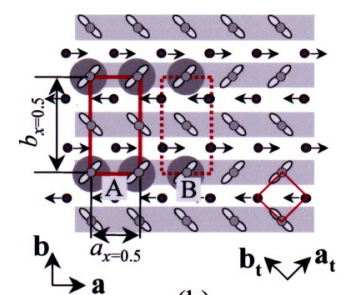

(b)

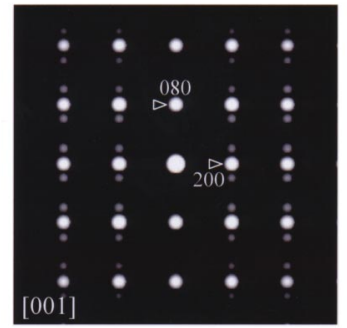

(c)
FIG. 1. (Color online) (a) The charge-ordered structural model of $\mathrm{La}_{2-2 x} \mathrm{Sr}_{1+2 x} \mathrm{Mn}_{2} \mathrm{O}_{7}$ at $x=0.5$, which is composed of $\mathrm{A}$ and $\mathrm{B}$ two kind layers. The $\mathrm{Mn}^{3+} \mathrm{O}_{6}$ and $\mathrm{Mn}^{4+} \mathrm{O}_{6}$ octahedra are shown in different colors. Its space group is Bbmm; (b) a [001] projection description of this structure (see the text for details) with solid and broken frames define the layers A and B unit cell positions, respectively; (c) the simulated ED pattern along the [001] zone axis (thickness $t=4 \mathrm{~nm}$ ). 
which gives rise to a sharp CMR transition. ${ }^{12}$ A variety of antiferromagnetic $(\mathrm{AF})$ and spin canted magnetic structures has been identified from $0.3<x<1.0 .^{18,22}$ When $x<0.45$, no long-range $\mathrm{CO}$ structure was observed, while a long-range CO structure was observed at $x=0.5 .^{14,15,17}$ Recently the charge ordering phenomena were also recorded at higher doping levels in the range of $x=0.5-0.7,{ }^{19,20,22,24}$ while the $\mathrm{CO}$ structures remain unexplored. No long-range charge ordering was observed when $x>0.7 .{ }^{20}$ In this work, we study the $\mathrm{CO}$ structure of the bilayered manganite, for the first time, when $x$ deviates away from $x=0.5$ in order to have a better understanding of the charge ordering behavior of these materials.

The CO structure at $x=0.5$ determined by Argyriou et $a l .{ }^{17}$ is shown in Fig. 1(a). It is an orthorhombic structure, with a space group of Bbmm (No. 63) and lattice parameters of $a=0.5443, b=1.0194$, and $c=1.9816 \mathrm{~nm}$. Two kinds of layers, labeled as layers A and B, respectively, in Fig. 1(a), can be identified from this structure. Each of them contains double $\mathrm{MnO}_{6}$ sheets. This structure can be more simply described by the [001] layer projection as shown in Fig. 1(b), where only $\mathrm{Mn}$ sites are shown while $\mathrm{La}^{3+} / \mathrm{Sr}^{2+}$ and $\mathrm{O}^{2-}$ sites are not depicted. Solid circle symbols denote the $\mathrm{Mn}^{4+}$ site, and the dumbbell symbols $\mathrm{Mn}^{3+}$ (the $\mathrm{Mn}^{3+}$ background is shadowed). The solid rectangle defines the atomic positions of the layer A, and the broken rectangles, layer B. Since the parent phase is a body-centered tetragonal structure with space group of $14 / \mathrm{mmm}^{13}$ (a unit cell of the parent phase with basis vectors $\mathbf{a}_{\mathrm{t}}$ and $\mathbf{b}_{\mathrm{t}}$ is outlined at the lower-right corner; the subscript $t$ denotes the tetragonal parent phase), the $\mathrm{Mn}$ sites of the layer B locate at the center of the Mn square network of the layer A, i.e. and the layer B is shifted by $(1 / 2) a$ with respect to the layer A. Note that the layer B is in fact $(1 / 2) c$ away from the layer A, while for convenience they are drawn together on the same plane. Arrows indicate the small displacement of $\mathrm{Mn}^{4+}$ from the parent phase (the neighboring $\mathrm{La}^{3+} / \mathrm{Sr}^{2+}$ and $\mathrm{O}^{2-}$ also have the same direction displacements but are not shown here). As decorated with the gray disks, it is a side $B$-centered lattice. Besides, this structure has the symmetry elements of axial glide $b[100]$, mirror $m[010]$ and mirror $m[001]$. From this structure at $x=0.5$, we will construct $\mathrm{CO}$ structures at higher doping levels with larger unit cells. These structures have different orderings in the $a-b$ plane, while they remain the same A and B layered structure. Figure 1(c) is a simulated electron diffraction (ED) pattern along the [001] zone axis, which exhibits superlattice reflections at $1 / 4$ positions of the fundamental ones, consistent with the experimental observations. ${ }^{14,15}$ Simulated ED patterns with superlattice reflections at $1 / 5$ and $1 / 6$ positions, at $x=0.6$ and $x=0.67$, respectively, will also be present according to our structural models given in this paper, and it will be shown that the superstructure at $x=0.6$ is indeed a novel face-centered one.

\section{EXPERIMENTAL PROCEDURES AND METHODS}

Single-phased samples were synthesized through chemical reactions and followed by annealing, for details see the previous report. ${ }^{22}$ Thin-foil transmission electron microscopy
TABLE I. Parameters for modeling possible charge-ordered structures of $\mathrm{La}_{2-2 x} \mathrm{Sr}_{1+2 x} \mathrm{Mn}_{2} \mathrm{O}_{7} . x=n /(m+n)$.

\begin{tabular}{|c|c|c|c|}
\hline $\begin{array}{l}\text { Doping } \\
\text { level, } x\end{array}$ & $\begin{array}{c}\text { Number } \\
\text { of } \mathrm{Mn}^{3+} / \mathrm{Mn}^{4+}, \\
m / n\end{array}$ & $\begin{array}{c}\text { Superlattice } \\
\text { reflection } \\
\text { position, } \\
1 / r\end{array}$ & $\begin{array}{l}\text { Modulation } \\
\text { vector }^{\mathrm{a}} \\
\mathbf{q}\end{array}$ \\
\hline 0.5 & $2 / 2$ & $1 / 4^{b}$ & $(1 / 4,1 / 4,0)$ \\
\hline 0.6 & $2 / 3^{c}(4 / 6)$ & $1 / 5^{b}$ & $(1 / 10,1 / 10,0)$ \\
\hline 0.67 & $2 / 4$ & $1 / 6^{b}$ & $(1 / 6,1 / 6,0)$ \\
\hline 0.71 & $2 / 5^{\mathrm{c}}(4 / 10)$ & $1 / 7$ & $(1 / 14,1 / 14,0)$ \\
\hline 0.75 & $2 / 6$ & $1 / 8^{b}$ & $(1 / 8,1 / 8,0)$ \\
\hline 0.78 & $2 / 7^{\mathrm{c}}(4 / 14)$ & $1 / 9$ & $(1 / 18,1 / 18,0)$ \\
\hline 0.8 & $2 / 8$ & $1 / 10$ & $(1 / 10,1 / 10,0)$ \\
\hline
\end{tabular}

${ }^{a}$ The discussion of $\mathbf{q}$ is given in this paper.

${ }^{b}$ These $1 / r$ positions have been confirmed experimentally (Refs. 19, 20, 22, and 24).

${ }^{c}$ When $n$ is odd, the cell should be doubled (doubled $m$ and $n$ are given in the brackets).

(TEM) specimens were prepared from polycrystalline bulk samples by mechanical thinning and finally ion milling with liquid $\mathrm{N}_{2}$ cooling to minimize the ion beam damage. The in-situ TEM work was carried out using a Hitachi H-9000 equipped with a liquid-helium cold stage working at $300 \mathrm{kV}$. Modeling and simulations were performed using a MS Modeling and Cerius ${ }^{2}$ programs from Accelrys Inc., and a slightly modified MacTempas program. The following parameters are used for the simulation: voltage $E=300 \mathrm{kV}$, spherical aberration coefficient $C_{s}=2.8 \mathrm{~mm}$, half-width of the Gaussian spread of focus due to chromatic aberration $\Delta=20 \mathrm{~nm}$, and semiangle of beam convergence $q=1.0 \mathrm{mrad}$. Specific foil thickness $t$ and defocus value $\Delta f$ are given for each particular simulation.

In order to model the $\mathrm{CO}$ structure of $\mathrm{La}_{2-2 x} \mathrm{Sr}_{1+2 x} \mathrm{Mn}_{2} \mathrm{O}_{7}$, it is necessary to consider the compositional dependence first. Since $\mathrm{La}, \mathrm{Sr}$ and $\mathrm{O}$ have the valence of $+3,+2$ and -2 , respectively, the average valence of $\mathrm{Mn}$ is thus $3+x$ to balance the charges. Suppose there are $m$ rows of $\mathrm{Mn}^{3+}$ and $n$ rows of $\mathrm{Mn}^{4+}$ in a unit cell, the fractions of $\mathrm{Mn}^{3+}$ and $\mathrm{Mn}^{4+}$ are thus $m /(m+n)$ and $n /(m+n)$, respectively, thus we obtain

$$
3 \times \frac{m}{m+n}+4 \times \frac{n}{m+n}=3+x ;
$$

hence

$$
x=\frac{n}{m+n} .
$$

Since $m$ and $n$ should be two integrals and $m$ should be even since $\mathrm{Mn}^{3+}$ should be paired, one can find out the dependence of $x$ with $m$ and $n$ from the above equation. Table I lists some of the results from $x=0.5$ up to 0.8 . As will be demonstrated when $n$ is odd as for $x=0.6$, the unit cell should be doubled; so both $m$ and $n$ should be doubled, as shown in the brackets of the table. The expected superlattice reflection 


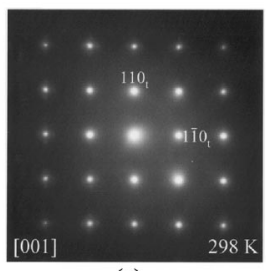

(a)

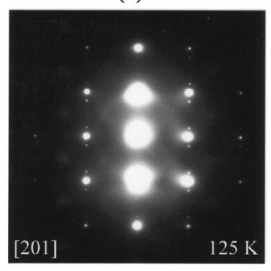

(d)

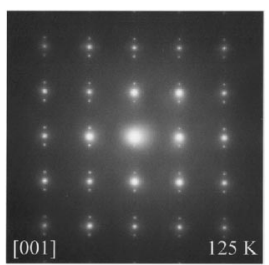

(b)

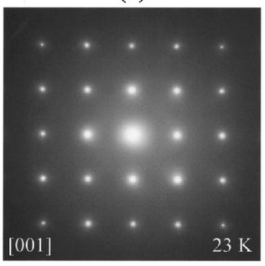

(e)

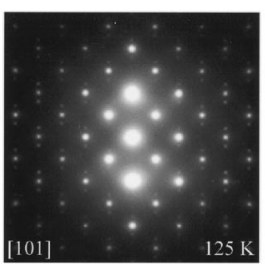

(c)
FIG. 2. In-situ ED patterns from a $\mathrm{La}_{2-2 x} \mathrm{Sr}_{1+2 x} \mathrm{Mn}_{2} \mathrm{O}_{7}(x=0.6)$ crystal at a temperature of (a) $298 \mathrm{~K}$ [001]; (b) $125 \mathrm{~K}$ [001]; (c) 125 $\mathrm{K}$ [101]; (d) $125 \mathrm{~K}$ [201]; and (e) $49 \mathrm{~K}$ [001].

position $1 / r$ is also given, where $r$ is the planar spacing ratio of the superlattice reflection to the fundamental one, e.g. in Fig. 1(c), $r=4$. Those marked with $* *$ in the table have been experimentally observed. It is clear that $1 / r$ is related to the total Mn number $m+n$, so $1 / r \sim x$ has a linear relationship. If one chooses higher integral numbers other than those listed in the table, larger unit cells, with shorter $1 / r$ superlattice positions, will be obtained. The possible modulation vector $\mathbf{q}$ for each $x$ is also listed in the table, which will be discussed in the following text.

\section{RESULTS AND DISCUSSIONS}

Figure 2 shows in-situ ED patterns from a crystal with $x$ $=0.6$ at different temperatures. At the room temperature (a), the reflections are consistent with the space group of $I 4 / \mathrm{mmm}$ of the tetragonal parent phase. However, extra superlattice reflections appear at the lower temperatures as a result of the charge ordering, as shown in Fig. 2(b) taken at $125 \mathrm{~K}$. It is seen that the extra reflections appear at the $1 / 5$ positions of the fundamental ones, while at $x=0.5$ at the $1 / 4$ positions. ${ }^{14,15}$ Figures 2(c) and 2(d) are the [101] and [201] zone axis diffraction patterns obtained by tilting from the [001] along the $(110)_{\mathrm{t}}^{*}$ reciprocal vector for about $13.9^{\circ}$ and $30.1^{\circ}$, respectively (the calculated angle between [001] and [101] is $15.4^{\circ}$, and between [001] and [201] is $28.8^{\circ}$ ), where the $1 / 5$ position superlattice reflections are still visible. At the even lower temperature $23 \mathrm{~K}$, the superlattice reflections disappear [Fig. 2(e)]. Note that the CO structure was found stable at this low temperature in a bulk sample as reported very recently, ${ }^{24}$ which implies that the disappearance of the superlattice reflections from the ED patterns is probably due to the TEM sample thin-film effect, where less strain is present to hinder the transition from the $\mathrm{CO}$ to a charge disordered A-type antiferromagnetic phase. ${ }^{18,22}$ However, a comparison of the superlattice reflection intensities and transport measurements as a function of temperature indicates that the charge ordering is responsible for the pronounced change in electrical resistance. This fact is consis-

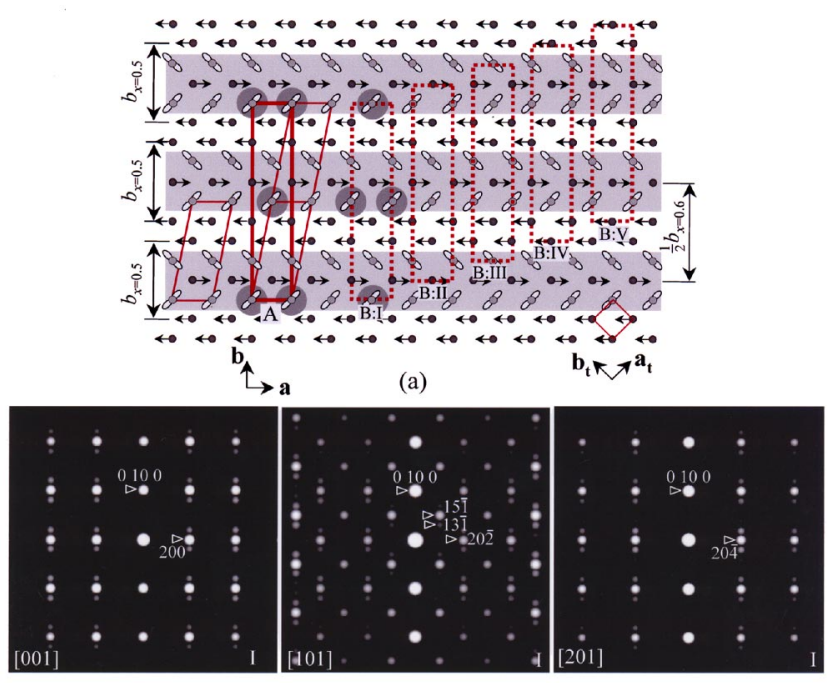

(b)
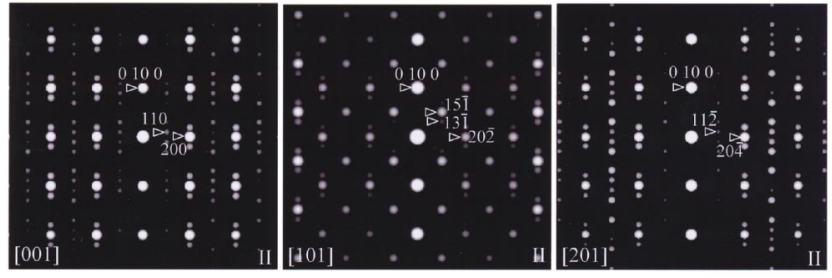

(c)

FIG. 3. (Color online) CO structure for $x=0.6$ The solid rectangle defines the atomic positions of layer A, and the five broken rectangles, as marked with $\mathrm{I}-\mathrm{V}$, possible positions of layer $\mathrm{B}$. The structure with the planar cell I as layer B (model I) has the facecentered symmetry (the space group is $F 2 \mathrm{~mm}$ ), while models II-V only $C$-centering symmetry. Simulated ED patterns from models I and II along [001], [101] and [201] are shown in (b) and (c), respectively (all at thickness $t=4 \mathrm{~nm}$ ).

tent with bulk sample measurements by the neutron diffraction. ${ }^{24}$

It is known that at $x=0.5$, there are equal amounts of $\mathrm{Mn}^{3+}$ and $\mathrm{Mn}^{4+}(m / n=1) \cdot{ }^{17}$ However, in the present case, $x=0.6$, the ratio of $m / n$ is $2 / 3$ (Table I). Therefore, to consider the $\mathrm{CO}$ structure, two rows of $\mathrm{Mn}^{3+} \mathrm{O}_{6}$ and three rows of $\mathrm{Mn}^{4+} \mathrm{O}_{6}$ are used to construct the structural unit. No matter how these rows are assembled, the configuration is always unique, i.e., the two $\mathrm{Mn}^{3+} \mathrm{O}_{6}$ rows are separated by one and two rows of $\mathrm{Mn}^{4+} \mathrm{O}_{6}$ alternatively, as shown in Fig. 3(a). No other possible assemblies can be obtained. This is a typical bi-stripe model due to the presence of bi-stripes of $\mathrm{Mn}^{3+} \mathrm{O}_{6}$, as highlighted with a shadow background under $\mathrm{Mn}^{3+}$ to guide the eye, rather than the Wigner-crystal model in which only uni-stripes of $\mathrm{Mn}^{3+} \mathrm{O}_{6}$ are present. ${ }^{6-10}$ A planar unit cell of the layer A [first double $\mathrm{MnO}_{6}$ layer, refer to Fig. $1(\mathrm{a})$ ] with five rows is outlined by a parallelogram at the lower left side of Fig. 3(a). However, since the upper edge is shifted by exactly half edge length $a$ with respect to the lower edge, a doubled cell should be chosen as the Bravais lattice to exhibit this base $C$-centering symmetry, as outlined by a bold rectangle at the right side of the parallelogram. The decorating gray disks highlight the $C$-centering symmetry. However, for the layer B, there are five possible positions, as 
shown by broken rectangles marked from I to $\mathrm{V}$, respectively (the structural models with these planar cells as the layer B are thus accordingly named as models I to $\mathrm{V}$, respectively). The planar cell I is obtained by shifting the planar cell of the layer A along the $[1 \overline{1} 0]_{\mathrm{t}}$ direction, while planar cells II-V along the $[110]_{\mathrm{t}}$, direction but for different magnitudes. It is seen that the planar cell I has both the side $A$ - and $B$-centering symmetries. Therefore, in total, the model I has a face $F$-centering symmetry. The possible space group given by the modeling programs is $F 2 \mathrm{~mm}$ (No. 42 ), with symmetry elements of 2-fold rotation 2[100], mirror $m[010]$ and mirror $m[001]$ that are evident in the drawing. In fact, the structural unit of the $x=0.5 \mathrm{CO}$ structure can be recognized, as indicated at the left side of Fig. 3(a). Thus this structure is indeed assembled from the building blocks of the $x=0.5$ phase along the modulation direction, with $\mathrm{Mn}^{4+} \mathrm{O}_{6}$ rows connecting together by but shifted by $1 / 2 a$. For the simulation, we adopt the same displacements of $\mathrm{Mn}^{4+}, \mathrm{La}^{3+} / \mathrm{Sr}^{2+}$ and $\mathrm{O}^{2-}$ from the parent phase at the corresponding positions of the $x=0.5$ phase. ${ }^{17}$ The other planar cells II-V do not have any side centering, so the models II-V only have the $C$-centering. Simulated ED patterns from the models I and II are shown in Figs. 3(b) and 3(c), respectively, both along the [001], [101] and [201] zone axes. Because the model I has the $F$-centering, the $(h k l)$ appear only when $h, k$ and $l$ are all even or all odd. Both the geometry and intensities of the superlattice reflections in the simulated ED patterns are consistent with the experimental patterns in Fig. 2 (in the experimental patterns, double reflections are possible). Simulations of other ED patterns obtained by tilting also support this structural model. Since the lattice parameter $b$ of the supercell is indeed ten times longer than the $d_{110}$ spacing of the parent phase, the modulation vector is thus deduced as $\mathbf{q}$ $=(1 / 10,1 / 10,0)$, although the superlattice reflections appear at the $1 / 5$ positions as the $(00 l)$ reflections with odd $l$ are forbidden due the systematic absence. However, in the previous studies, ${ }^{19,24}$ the modulation vector was reported as $\mathbf{q}$ $=(1 / 5,1 / 5,0)$ by the appearance of $1 / 5$ position superlattice reflections while the $F$-centering was not considered. Note that linear relations of $\mathbf{q} \sim x$ were reported for bilayered ${ }^{19,27}$ as well as single-layered ${ }^{25,26}$ manganites. However, in the structural model in Fig. 3(a), $m / n=2 / 3$, since the total Mn number 5 is odd, the unit cell is doubled, and thus $\mathbf{q}$ should be $(1 / 10,1 / 10,0)$ instead of $(1 / 5,1 / 5,0)$. Similarly, in the case of $x=0.71, m / n=2 / 5$, there are seven Mn rows (see Table I); if this phase exists, possible $F$-centered structures can also be constructed for it (see the Appendix), which contain $14 \mathrm{Mn}$ rows in the supercell with $\mathbf{q}=(1 / 14,1 / 14,0)$ although the superlattice reflections appear at the $1 / 7$ positions. The situation is the same for $x=0.78$ with nine $\mathrm{Mn}$ rows. It seems that at different doping levels, as the superlattice reflection position $1 / r$ is related to $(m+n), 1 / r \sim x$ keeps a linear relationship; while $q$ is related to the supercell dimension so $q \sim x$ does not have such a linear relationship. When the composition deviates slightly away from $x=0.6$, the modulation may change its character to incommensurate as experimentally observed. ${ }^{19,24}$ Such a change can result from the modification of the basic structure studied here. In fact, in the previous TEM studies by Wang et $a l{ }^{10}$ in
$\mathrm{La}_{0.33} \mathrm{Ca}_{0.67} \mathrm{MnO}_{3}$, possibly due to the chemical composition of the local studying areas deviated away from the ideal composition $x=2 / 3$, the modulated structure they observed was incommensurate with $\mathbf{q}=(0.284,0, \xi)(|\xi|=0.010)$ rather than the commensurate Wigner-crystal structure with $\mathbf{q}$ $=(1 / 3,0,0$,$) , which implies that it is indeed an incommen-$ surately modified Wigner-crystal structure. Further studies are needed for the incommensurate structures with $x$ values located between the listed ones in Table I. Because model II only has the $C$-centering, the $(h k l)$ reflection condition is that $h+k$ is even, so extra reflection rows appear in the simulated patterns along [001] and [201] zone axes in Fig. 3(c). The models III-V give similar reflection geometry as Fig. 3(c). It should be pointed out that if one starts with more $\mathrm{MnO}_{6}$ rows to construct the model instead of the five rows in Fig. 3(a), infinite models can be obtained but the simulated results do not fit the experimental data. The unit cell in Fig. 3(a) indeed contains ten rows of $\mathrm{MnO}_{6}$. If one starts with ten rows of $\mathrm{MnO}_{6}$, besides the bi-stripe model in Fig. 3(a), tri- and quadstripe models can be obtained, however, their simulated ED patterns exhibit superlattice reflections at the $1 / 10$ positions.

An overview experimental HREM image of the CO structure observed at $125 \mathrm{~K}$ is shown in Fig. 4(a). Periodical dark fringes, with a spacing of $1.3 \mathrm{~nm}$, are observed, as marked with some arrowheads on the left side of the image. The inserted Fourier transformation pattern exhibits extra spots at the $1 / 5$ positions of the fundamental spots, as shown by arrowheads. Figure 4(b) is a magnified noised-filtered image, which displays the structural modulation from the variation of image spot intensities. A simulated image by the $F$-centered structure model in Fig 3(a) is inserted in Fig. 4(b) for a comparison.

From the HREM images recorded at different areas and at different defocus values, two typical image features are revealed, as shown on the left-half sides of Figs. 5(a) and 5(b), respectively. In Fig. 5(a), the image intensity along the vertical direction profiles a feature with three strong and two weak lines (we denote this feature as $3 / 2 / 3 / 2$; the bold font stands for the strong line), while in Fig. 5(b), four strong and one weak line $(4 / 1 / 4 / 1)$. For thicker samples, due to the invalidity of the weak-phase object approximation, quantitative image simulation based on the dynamic diffractiontheory is essential in interpreting the experimental HREM images. ${ }^{28}$ The image simulation shows that these image features can be explained using the $F$-centered structural model in Fig. 3(a). As shown in Fig. 5(c), although the image appears differently at different $t$ and $\Delta f$, the both $\mathbf{3} / 2 / \mathbf{3} / 2$ and 4/1/4/1 features are seen in the simulations. Two simulated images at $t=4 \mathrm{~nm}$ but two different defocus values are shown at the right-half sides of Figs. 5(a) and 5(b), respectively, for comparison. A quantitative comparison of the intensity profiles shows good matches between the experimental and simulated images. None of the models II-V in Fig. 3(a) gives satisfied simulated images with these image features over a wide range of imaging conditions, even when the beam or specimen tilts are considered. ${ }^{29}$

Further, we discuss the $\mathrm{CO}$ structure at the doping level of $x=0.67$. The experimental data have been reported previously that were acquired by the same type of microscope $\mathrm{H}-9000$ as we used here. ${ }^{19}$ In the case of $x=0.67$, the ratio of 


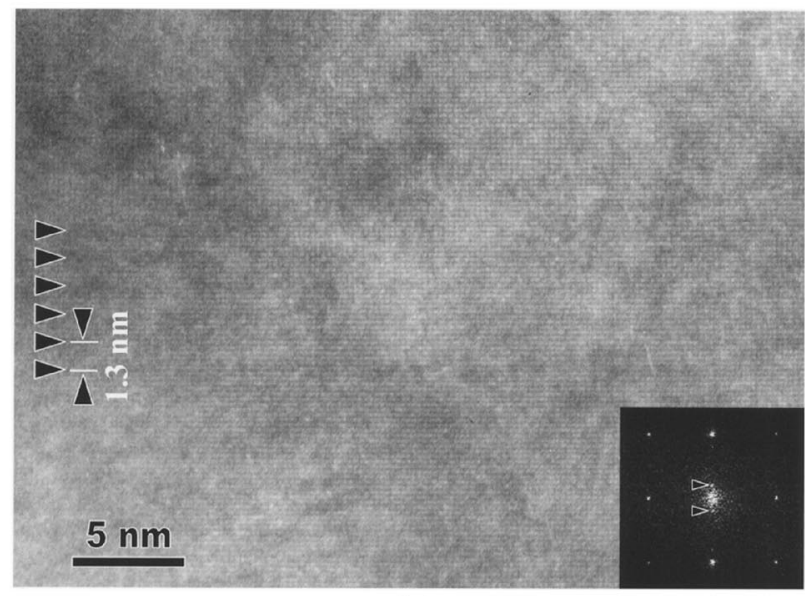

(a)

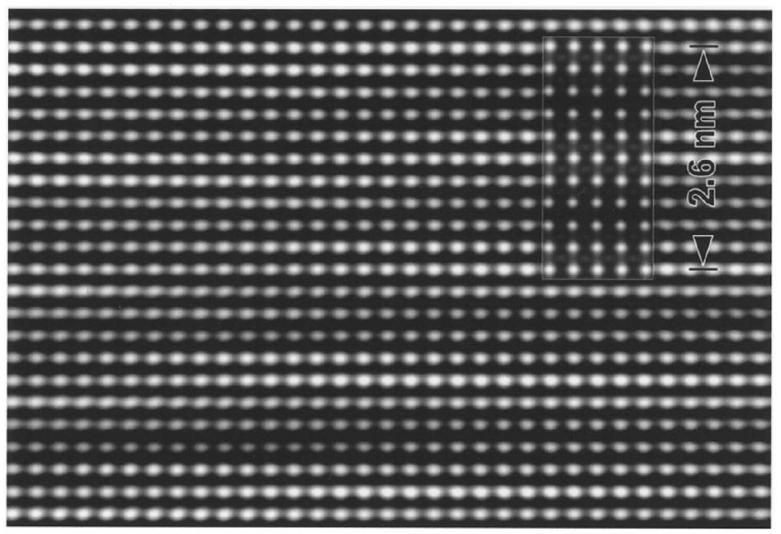

(b)

FIG. 4. (a) HREM image of the CO phase of $x=0.6$ taken at 125 $\mathrm{K}$, with a Fourier transformation pattern inserted; (b) noise filtered image, with a simulated image $(t=4 \mathrm{~nm}, \Delta f=-190 \mathrm{~nm})$ inserted according to the face-centered structure in Fig. 3(a).

$m / n$ is $2 / 4$ (see Table I). There are only two possible models as shown in Figs. 6(a) and 6(b), which are named as the Wigner-crystal or the bi-stripe models, respectively. Both of them have the $B$-centering symmetry, as decorated with the gray disks, by the selection of the layer B planar cells shifting along the $[1 \overline{1} 0]_{\mathrm{t}}$ as already demonstrated in Fig. 3. The possible space groups given by the modeling programs is Bbcm (No. 64) for Fig. 6(a), and B2mm (No. 38) for Fig. $6(b)$. Building blocks of $(1 / 2) b$ of the $x=0.6$ phase in Fig. 3(a) are found in the Wigner-crystal model in Fig. 6(a), while building blocks of the $x=0.5$ phase in the bi-stripe model in Fig. 6(b). Simulated electron diffraction patterns of both models exhibit $1 / 6$ position superlattice reflections as reported, ${ }^{19}$ as shown in Figs. 6(c) and 6(d), respectively. Therefore, we use the HREM image feature to discriminate between them.

The HREM image taken by Li et al. ${ }^{19}$ displays clearly a feature with three strong and three weak lines $(\mathbf{3} / 3)$, as shown on the left-half side of Fig. 7(a) (after noise elimination). The band spacing is about $1.6 \mathrm{~nm}$. Image simulations of the two models in Figs. 6(a) and 6(b) show different image features. The Wigner-crystal model in Fig. 6(a) gives

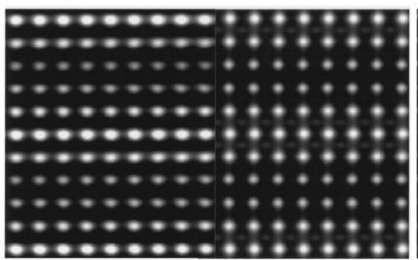

(a)

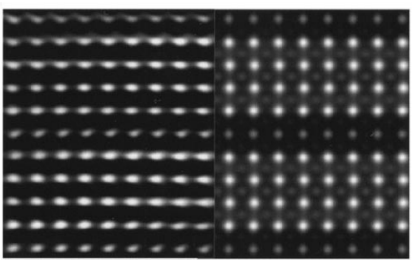

(b)

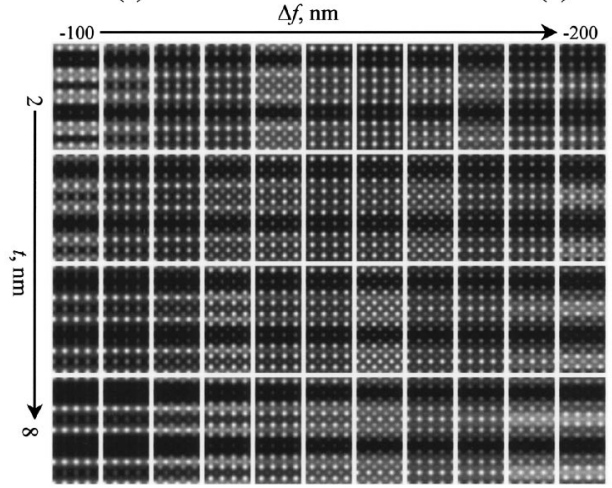

(c)

FIG. 5. (a), (b) Two typical HREM images from the sample with $x=0.6$ shown on the left-half side, and simulated images by the $F$-centered model in Fig. 3(a) shown on the right-half side [both $t$ $=4 \mathrm{~nm}$, but $\Delta f=-190 \mathrm{~nm}$ for (a) and $\Delta f=-150 \mathrm{~nm}$ for (b)]; (c) imaging simulation over wide imaging conditions (images are shown in $2 a \times 1 b)$.
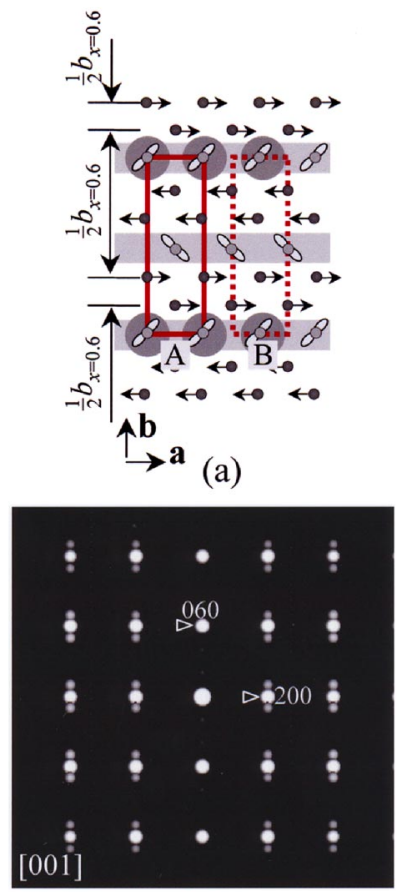

(c)

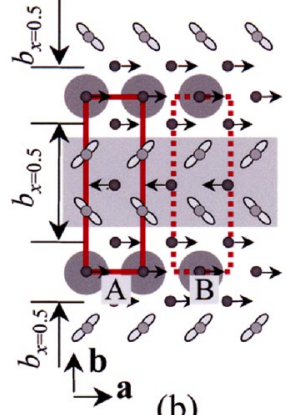

(b)

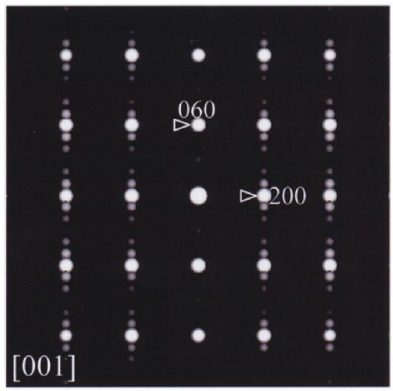

(d)
FIG. 6. (Color online) Two possible CO models for $x=0.67$ : (a) the Wigner-crystal model (the space group is $B b c m$ ), and (b) the bi-stripe model (the space group is $B 2 \mathrm{~mm}$ ). Simulated ED patterns from them are shown below the models in (c) and (d), respectively (both $t=4 \mathrm{~nm}$ ). 


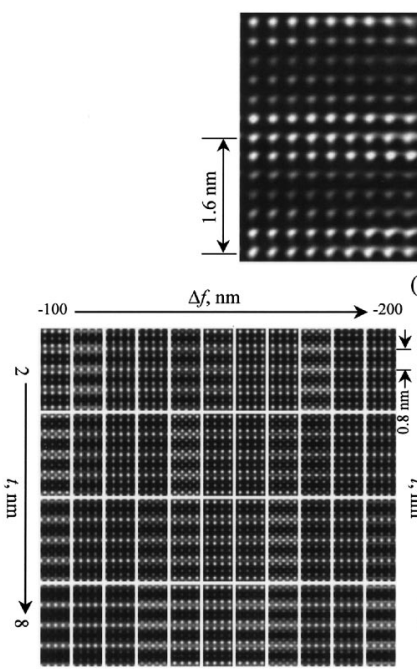

(b)

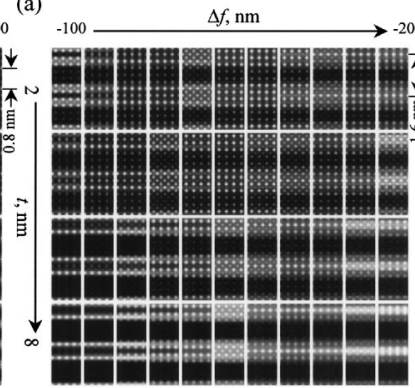

(c)
FIG. 7. The noise filtered image from the HREM image ( $x$ $=0.67$ ) taken by Li et al. (Ref. 19) is shown on the left-half side of (a), compared with the simulated image by the bi-stripe model in Fig. 6(b) at the right-half side $(t=2 \mathrm{~nm}, \Delta f=-130 \mathrm{~nm})$. Image simulation over wide imaging conditions according to the Wignercrystal model and the bi-stripe models are shown in (b) and (c), respectively (images are shown in $2 a \times 2 b$ ).

image features typically the alternative appearance of one strong and two weak lines $(1 / 2 / 1 / 2)$ or two strong and one weak lines $(2 / 1 / 2 / 1)$, i.e. no image stripes wider than two lines can be obtained by this model, as shown in Fig. 7(b). As a result, an image band only contains three rows with spacing of about $0.8 \mathrm{~nm}$. This is expected, since the $\mathrm{Mn}^{3+} \mathrm{O}^{6}$ and $\mathrm{Mn}^{4+} \mathrm{O}_{6}$ rows are evenly distributed in the Wignercrystal model in Fig. 6(a). However, intense wider image stripes can be obtained from the bi-stripe model, with image features of $\mathbf{3 / 3}, \mathbf{4 / 2}$ or $\mathbf{5 / 1}$, as shown in Fig. 7(c). In this case, an image band contains six rows, with spacing of about

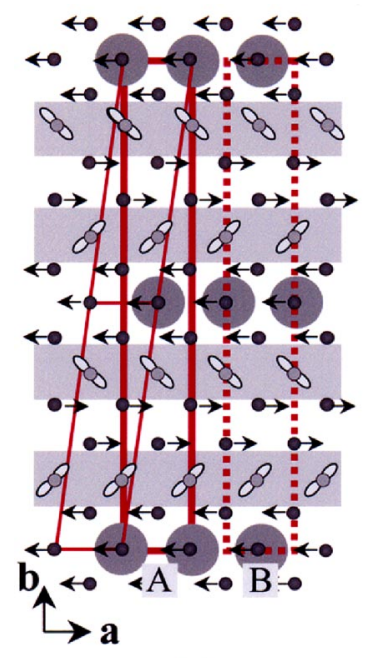

(a)

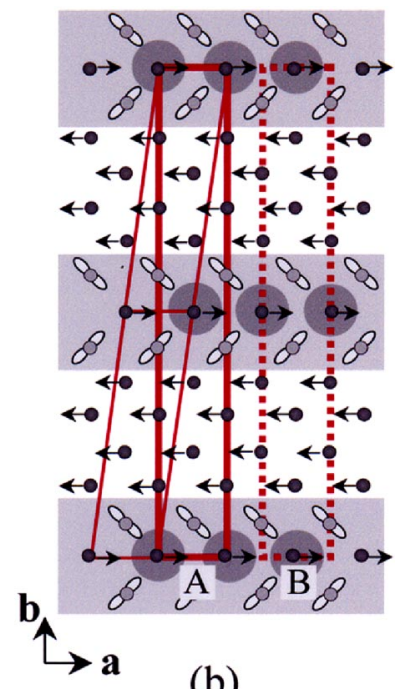

(b)
FIG. 8. (Color online) Two possible $F$-centered CO structures for $x=0.71$ : (a) Wigner-crystal model; (b) bi-stripe model.
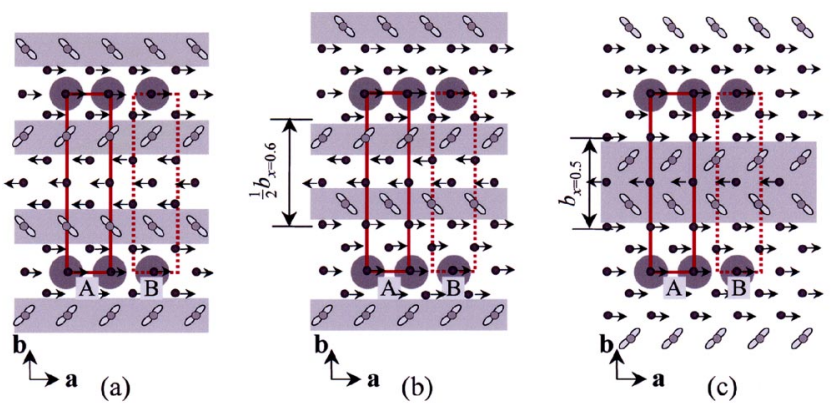

FIG. 9. (Color online) Three possible CO structures for $x$ =0.75: (a) and (b) Wigner-crystal models; (b) the bi-stripe model.

$1.6 \mathrm{~nm}$ that is consistent with the observed HREM image. A simulated image is shown on the right-half side on Fig. 7(a). Such a 3/3 image feature cannot be obtained from the Wigner-crystal structural model over a wide range of imaging conditions including the specimen or beam tilts. ${ }^{29}$ It is noted that the HREM image from $\mathrm{La}_{2-2 x} \mathrm{Ca}_{1+2 x} \mathrm{Mn}_{2} \mathrm{O}_{7}$ sample also exhibits the $3 / 3$ and $\mathbf{4} / 2$ features from this type of CO structure (1/6 position superlattice reflections), ${ }^{27} \mathrm{im}$ plying that the bi-stripe model in Fig. 6(b) may also apply for the $\mathrm{La}_{2-2 x} \mathrm{Ca}_{1+2 x} \mathrm{Mn}_{2} \mathrm{O}_{7}$ system. However in the $\mathrm{La}_{2-2 x} \mathrm{Ca}_{1+2 x} \mathrm{Mn}_{2} \mathrm{O}_{7}$ system, the fact that the $1 / 6$ position superlattice reflection structure was formed even at the $x$ $=0.6$ doping level ${ }^{27}$ suggests that this $1 / 6$ position superstructure may be more stable than the $1 / 5$ position superstructure in Fig. 3.

\section{CONCLUSIONS}

In summary, we have extended our understanding of the charge-ordered structure in the bilayered manganite $\mathrm{La}_{2-2 x} \mathrm{Sr}_{1+2 x} \mathrm{Mn}_{2} \mathrm{O}_{7}$ from $x=0.5$ to higher doping levels and have recognized two new basic charge-ordered superstructures based on the electron microscopic studies, at the doping level of $x=0.6$ and $x=0.67$, respectively. The charge-ordered phase at $x=0.6$ has a face-centered structure, with $\mathbf{q}$ $=(1 / 10,1 / 10,0)$, and the charge-ordered phase at $x=0.67$ has $\mathbf{q}=(1 / 6,1 / 6,0)$. In both cases, bi-stripe models have been suggested.

\section{ACKNOWLEDGMENTS}

The authors thank Mr. Edward Ryan for technical assistance in using the liquid-helium cold stage on H-9000 in the Electron Microscopy Center of the Argonne National Laboratory. Dr. Lisa M. Pérez, Laboratory for Molecular Simulation, Department of Chemistry, Texas A\&M University, is also acknowledged for assistance in using the modeling programs. This work was supported by the U.S. Department of Energy, Division of Basic Energy Sciences-Material Sciences, under Contract, No. W-31-109-Eng-38.

\section{APPENDIX: POSSIBLE CHARGE-ORDERED STRUCTURES AT OTHER DOPING LEVELS}

Table I lists seven possible $\mathrm{CO}$ structure parameters from $x=0.5$ to 0.8 . The $x=0.5,0.6$ and $0.67 \mathrm{CO}$ structures have 
been studied in this paper. Here we give two more examples at $x=0.71$ and 0.75 , although so far no experimental data are available to confirm their existence or their particular model.

When $x=0.71, m / n=2 / 5$ (see Table I). If one uses two rows of $\mathrm{Mn}^{3+} \mathrm{O}_{6}$ and five rows of $\mathrm{Mn}^{4+} \mathrm{O}_{6}$ to construct the unit cell, there are only two possibilities, as shown in Figs. 8(a) and 8(b), respectively. In Fig. 8(a), the single $\mathrm{Mn}^{3+} \mathrm{O}_{6}$ stripes are separated by two or three rows of $\mathrm{Mn}^{4+} \mathrm{O}_{6}$, so it is a Wigner-crystal model, while in Fig. 8(b), bi-stripes of $\mathrm{Mn}^{3+} \mathrm{O}_{6}$ are isolated by four rows of $\mathrm{Mn}^{4+} \mathrm{O}_{6}$, so it is a bi-stripe model. Similar to the case of $x=0.6$ in Fig. 3, the unit cell is doubled, therefore, both of them are $F$-centered. If one starts with four rows of $\mathrm{Mn}^{3+} \mathrm{O}_{6}$ and ten rows of $\mathrm{Mn}^{4+} \mathrm{O}_{6}$, many other assemblies can be obtained, but they are not $F$-centered.

At $x=0.75, m / n=2 / 6$ (see Table I). They are three possible assemblies, as shown in Figs. 9(a)-9(c), respectively. In Figs. 9(a) and $9(\mathrm{~b})$, single $\mathrm{Mn}^{3+} \mathrm{O}_{6}$ stripes are separated by a different number $\mathrm{Mn}^{4+} \mathrm{O}_{6}$ rows, so both of them can be named a the Wigner-crystal model. Figure 9(c) is a bi-stripe model by the presence of bi-stripe $\mathrm{Mn}^{3+} \mathrm{O}_{6}$. All these three models are $B$-centered.

Other possible CO structures at $x=0.78$ and 0.8 can be deduced in a similar way.
*Corresponding author. Electronic address: luo@mic.tamu.edu

${ }^{1}$ J. B. Goodenough, Phys. Rev. 100, 564 (1955).

${ }^{2}$ P. G. Radaelli, D. E. Cox, M. Marezio, and S-W. Cheong, Phys. Rev. B 55, 3015 (1999).

${ }^{3}$ S. Mori, C. H. Chen, and S.-W. Cheong, Nature (London) 392, 473 (1998)

${ }^{4}$ S. Mori, C. H. Chen, and S.-W. Cheong, Phys. Rev. Lett. 81, 3972 (1998).

${ }^{5}$ C. H. Chen, S. Mori, and S.-W. Cheong, J. Phys. IV 9, 307 (1999).

${ }^{6}$ M. T. Fernández-Díaz, J. L. Martínez, J. M. Alonso, and E. Herrero, Phys. Rev. B 59, 1277 (1999).

${ }^{7}$ P. G. Radaelli, D. E. Cox, L. Capogna, S.-W. Cheong, and M. Marezio, Phys. Rev. B 59, 14440 (1999).

${ }^{8}$ E. P. Wigner, Phys. Rev. 46, 1002 (1934).

${ }^{9}$ C. H. Chen, S-W. Cheong, and H. Y. Hwang, J. Appl. Phys. 81, 4326 (1997).

${ }^{10}$ R. Wang, J. Gui, Y. Zhu, and A. R. Moodenbaugh, Phys. Rev. B 61, 11946 (2000).

${ }^{11}$ Y. Moritomo, A. Asamitsu, H. Kuwahara, and Y. Tokura, Nature (London) 380, 141 (1996).

${ }^{12}$ T. Kimura, Y. Tomioka, H. Kuwahara, A. Asamitsu, M. Tamura, and Y. Tokura, Science 274, 1698 (1996).

${ }^{13}$ J. F. Mitchell, D. N. Argyriou, J. D. Jorgensen, D. G. Hinks, C. D. Potter, and S. D. Bader, Phys. Rev. B 55, 63 (1997).

${ }^{14}$ J. Q. Li, Y. Matsui, T. Kimura, and Y. Tokura, Phys. Rev. B 57, R3205 (1998).

${ }^{15}$ T. Kimura, R. Kumai, Y. Tokura, J. Q. Li, and Y. Matsui, Phys. Rev. B 58, 11081 (1998).

${ }^{16}$ D. N. Argyriou, J. F. Mitchell, P. G. Radaelli, H. N. Bordallo, D. E. Cox, M. Medarde, and J. D. Jorgensen, Phys. Rev. B 59,
8695 (1999).

${ }^{17}$ D. N. Argyriou, H. N. Bordallo, B. J. Campbell, A. K. Cheetham, D. E. Cox, J. S. Gardner, K. Hanif, A dos Santos, and G. F. Strouse, Phys. Rev. B 61, 15269 (2000).

${ }^{18}$ C. D. Ling, J. E. Millburn, J. F. Mitchell, D. N. Argyriou, J. Linton, and H. N. Bordallo, Phys. Rev. B 62, 15096 (2000).

${ }^{19}$ J. Q. Li, C. Dong, L. H. Liu, and Y. M. Ni, Phys. Rev. B 64, 174413 (2001).

${ }^{20}$ J. Dho, W. S. Kim, H. S. Choi, E. O. Chi, and N. H. Hur, J. Phys.: Condens. Matter 13, 3655 (2001).

${ }^{21}$ B. J. Campbell, R. Osborn, D. N. Argyriou, L. Vasiliu-Doloc, J. F. Mitchell, S. K. Sinha, U. Ruett, C. D. Ling, Z. Islam, and J. W. Lynn, Phys. Rev. B 65, 014427 (2001).

${ }^{22}$ J. F. Mitchell, C. D. Ling, J. E. Millburn, D. N. Argyriou, A. Berger, M. Medarde, D. Miller, and Z. P. Luo, Appl. Phys. A: Mater. Sci. Process. 74, S1776 (2002).

${ }^{23}$ S. B. Wilkins, P. D. Spencer, T. A.W. Beale, P. D. Hatton, M. v. Zimmermann, S. D. Brown, D. Prabhakaran, and A. T. Boothroyd, Phys. Rev. B 67, 205110 (2003).

${ }^{24}$ B. J. Campbell, D. N. Argyriou, J. F. Mitchell, R. Osborn, B. Ouladdiaf, and C. D. Ling, Phys. Rev. B 69, 104403 (2004).

${ }^{25}$ S. Larochelle, A. Mehta, N. Kaneko, P. K. Mang, A. F. Panchula, L. Zhou, J. Arthur, and M. Greven, Phys. Rev. Lett. 87, 095502 (2001).

${ }^{26}$ Z. J. Feng, C. Dong, Z. C. Li, and J. Q. Li, Chin. Phys. Lett., 21, 187 (2004)

${ }^{27}$ J. Q. Li, C. Q. Jin, and H. B. Zhao, Phys. Rev. B 64, 020405(R) (2001).

${ }^{28}$ J. C. H. Spence, High-Resolution Electron Microscopy, 3rd ed. (Oxford University Press, Oxford, 2003).

${ }^{29}$ H. W. Zandbergen and J. Jansen, Ultramicroscopy 80, 59 (1999). 